\title{
A Rumor Spreading Model considering the Cumulative Effects of Memory
}

\author{
Yi Zhang ${ }^{1,2}$ and Jiuping $X u^{1}$ \\ ${ }^{1}$ Business School, Sichuan University, Chengdu 610064, China \\ ${ }^{2}$ Department of Mathematics, Yibin University, Yibin 644007, China \\ Correspondence should be addressed to Jiuping Xu; xujiuping@scu.edu.cn
}

Received 25 July 2014; Accepted 26 September 2014

Academic Editor: Antonia Vecchio

Copyright (c) 2015 Y. Zhang and J. Xu. This is an open access article distributed under the Creative Commons Attribution License, which permits unrestricted use, distribution, and reproduction in any medium, provided the original work is properly cited.

\begin{abstract}
This paper proposes a rumor spreading model which examines how the memory effects rate changes over time in artificial network and a real social network. This model emphasizes a special rumor spreading characteristic called "the cumulative effects of memory." A function $p(t)$ reflecting the cumulative memory effects is established, which replaces the constant rate of memory effects in the traditional model. Further, rumor spreading model simulations are conducted with different parameters in three artificial networks. The results show that all the parameters but the initial memory rate of memory effects function have a significant impact on rumor spreading. At the same time, the simulation results show that the final size of the stiflers is sensitive to the average degree $\langle k\rangle$ when it is small but is not sensitive to $\langle k\rangle$ when the average degree is greater than a certain degree. Finally, through investigations on the Sina Microblog network, the numerical solutions show that the peak value and final size of the rumor spreading are much larger under a variable memory effects rate than under a constant rate.
\end{abstract}

\section{Introduction}

An old saying goes that rumors come true after being repeated a thousand times. In real life, if people are unable to distinguish authenticity, many rumors are deemed to be true after a large number of repetitions. When rumors are widely propagated, people tend to believe the rumor, especially if they lack timely real information. Because of the increased presence of online social networks, rumors are no longer spread by word of mouth over a small area but are spread amongst strangers in different regions and different countries, meaning that rumors are being spread faster and wider than ever before. This sustained and rapid spreading of rumors deepens people's impression about the veracity of the rumor and thus improves the credibility. Rumor spreading, therefore, has the ability to shape public opinion and lead to social panic and instability [1]. For example, the 2011 Tohoku nuclear leakage accidents caused a number of rumors in China. Rumors said that taking materials containing iodine could help ward off nuclear radiation, which led to the fact that many people rushed to purchase iodized salt.
Rumor spreading has attracted significant attention from researchers and, as a result, a great deal of research has been done on rumor spreading models. In the early stages, scholars borrowed from epidemic models to describe rumor spreading process [2-4]. A classical model is the SIR model, which studies the dynamic behavior of rumor spreading using an epidemic dynamics SIR model [5]. Based on this research, there have been many more rumor spreading models, most of which have been a variant of the SIR model [6-8]. With the development of network technology, many novel models have appeared inspired by empirical discoveries about network topology [7,9-11]. At the same time, researchers have started to consider the specific features of rumor spreading in their models. Dodds and Watts [12] studied the effects of limited memory on contagion. Zhao et al. $[13,14]$ proposed a rumor spreading model which considered remembering mechanisms in homogeneous and inhomogeneous networks.

However, most previous studies have discussed memory effects as a constant parameter in the model, but, in reality, people hear rumors many times and so have an accumulation 
of impressions about the rumors, which changes the probability as to when people become rumor spreaders. Therefore, memory effects have a strong time-dependency. Further, the remembering mechanisms can indicate repeatability, which affects the spreading characteristics of the rumor [13]. Even a small amount of memory can affect the rumor spread in small network sizes [15]. Lü et al. [16] proposed a model considered memory effects on information spread but did not construct a function of time and did not build spreading dynamics equations.

In the existing research on rumor propagation, there have been two main classification methods for the population. In the first, the population is divided into the three groups borrowed from the epidemic spreading classifications [5], those that are susceptible (have never heard the rumor), those who are infected (are spreading the rumor), and those who are recovered (have heard the rumor but do not spread it). These classifications have been adopted in most rumor spreading models. In the second classification, however, the population is divided into four groups. This classification adds a new group to the first classification, but this group has been interpreted differently in different studies. In some research, this additional group, which arises from the spreaders, is called the hibernators, those that have heard but forgotten the information [13]. In Lü's research [16], the additional group is called the known, those who are aware of the rumor but are not willing to transmit it as they are suspicious of its authenticity. An incubation class was suggested in [17], where it was proposed that a susceptible individual first goes through a latent period after being infected before becoming a spreader or a stifler, which is a similar idea to that of Lü. In this paper, we follow a four-group classification including incubation class, the unaware, the spreaders, the lurkers, and the stiflers.

In this paper, we study a rumor spreading model with variable rate which considers cumulative memory effects as a function of time, which is more in line with reality. The remainder of this paper is organized as follows. In Section 2, first, the rumor spreading model with a variable rate varying over time is described. Then, the mean-field equations are derived, and the dynamic analysis of the model is conducted. In Section 3, numerical simulations demonstrating the dynamics of the established model are conducted on regular networks, random networks, BA networks, and online social networks. We compare our model using different parameters and analyze the model's impact at different average degree of networks. Then, we make comparisons between a constant versus a variable rate on Sina Microblog. In Section 4, further discussion is presented. Finally, in Section 5, we conclude this paper and provide several avenues for further research.

\section{A Rumor Spreading Model with Variable Memory Rate}

In this model, we construct a function of time $t$ which reflects the accumulation of memory, the speed of memory change, and the importance of an event which triggers rumors. Then, with this function added as a parameter to a differential dynamic spread model, a new rumor propagation model is proposed.

2.1. Memory Effects Function. Consider a network with $N$ nodes and $E$ links representing the individuals and their interactions. At each time step, each individual is in one of the following four states:

(1) the unaware: this individual has not yet heard the rumor;

(2) the lurkers: this individual knows the rumor but is not willing to spread it because they require an active effort to discern the truth or falseness of the rumor;

(3) the spreaders: this individual knows this rumor and transmits it to all their contacts;

(4) the stiflers: this individual neither trusts the rumor nor transmits it.

People generally hear a rumor after many times, and therefore they get an accumulated impression about the rumor, which means that the probability that people become a spreader changes from "will never believe" to "believes." This can be described as the cumulative effect of memory, which affects the probability that an individual becomes a spreader from a lurker in the rumor spreading process. In information spreading theory, a function was established which reflected the probability that a person would approve the information at time $t$ after having received the news $m$ times [16]. This function is $P(m)=(\lambda-T) e^{-b(m-1)}+T$, where $\lambda=P(1)$ is the approving probability of the first receipt of the information and $T \in(0,1]$ is the upper bound of the probability indicating maximal approval probability. In fact, as $m$ is a function of time, we can transform this function into a function of time $t$; that is, the probability that a person approves the rumor at time $t$ can be denoted by a function of time $t$. From the following analysis, we determine a specific form for the function of time $t$.

First, we analyze the changing process of the lurkers. From a microcosmic point of view, lurkers do not automatically change their states at time step $t$. Some may become a stifler or a spreader, while others remain lurkers and may become stiflers or spreaders at a later time. We assume that the new lurkers at each time step have a part of the residuals which last until the end of the rumor spreading. This corresponds with the fact that there are always some people who take a long time to change their state in real life. From the above analysis, lurkers become spreaders at a variable probability, denoted by $p(t)$, and become stiflers at the rate of $p_{2}$, so we can determine the lurkers' process of change at each time step, as shown in Figure 1. In the following paragraph, we explain this function $p(t)$.

As the number of times the rumor is received, the probability that a individual agrees to the truth of the rumor grows and infinitely approaches a constant [16]. Thus, as time passes, the number of times the rumor is received for the residual lurkers gradually increases. Because the probability $p(t)$ that an individual becomes a spreader from 


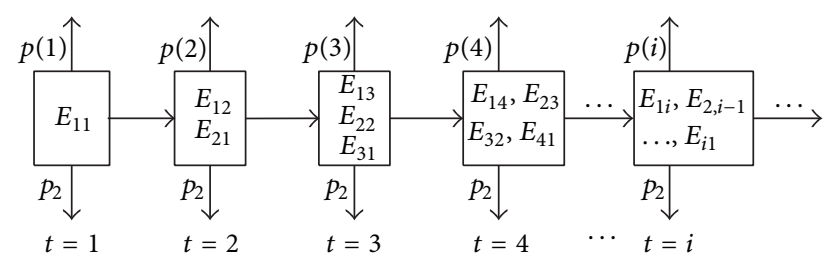

FigurE 1: The change of the lurker at each step. $E_{i 1}$ represents new lurkers at the $i$ th time step and $E_{i j}(j \neq 1)$ represents the $j$ th residual of $E_{i 1}$. The total number of lurkers is $E(i)=E_{i 1}+E_{i-1,2}+E_{i-2,3}+\cdots+$ $E_{2, i-1}+E_{1 i}$ at the $i$ th time step. For example, $E(3)=E_{31}+E_{22}+E_{13}$.

a lurker is a level that reflects the transformation probability of all lurkers, including the residual old lurkers and the new joined lurkers in each time step, as time passes, the probability increases gradually because of the cumulative effect of memory and infinitely approaches a constant when the accumulated memories achieve a certain degree.

In the following, we show how probability $p(t)$ becomes larger and infinitely approaches a constant. Figure 2 shows the probabilities that each part of the lurkers becomes spreaders. Firstly, we prove a lemma.

Lemma 1. In the rumor spreading process, there is a time point $t=m$, such that $p_{1, k} \leq p_{2, k} \leq p_{3, k} \leq \cdots \leq p_{i, k}(i, k=$ $1,2,3, \ldots, i+k \leq m)$ and $p_{j, 1} \leq p_{j, 2} \leq p_{j, 3} \leq \cdots \leq p_{j, h}(j, h=$ $1,2,3, \ldots, j+h \leq m)$ before $t=m$.

Proof. According to rumor spreading theory, at the beginning of the process, there is an increase in the number of spreaders as the rumor propagates, and, with the further spreading of the rumor, the number of spreaders reaches a peak and then decreases as time goes on. Finally, the number of spreaders is zero and the rumor spreading terminates.

Therefore, with an increase in the spreaders, the probability of the lurkers receiving the rumor increases gradually before the number of spreaders reaches a peak at least. We assume this time point to be $t=m$. That is, the probability that the lurkers become spreaders increases before $t=m$. Therefore, there is a time point $t=m$, such that $p_{1, k} \leq$ $p_{2, k} \leq p_{3, k} \leq \cdots \leq p_{i, k}(i, k=1,2,3, \ldots, i+k \leq m)$ and $p_{j, 1} \leq p_{j, 2} \leq p_{j, 3} \leq \cdots \leq p_{j, h}(j, h=1,2,3, \ldots, j+h \leq m)$ before $t=m$.

Because $p(t)$ is a level that reflects the transformation probability of all lurkers, including the residual old lurkers and the new joined lurkers in each time step, we prove that it has the following properties.

Theorem 2. With an increase in time, the probability $p(t)$ becomes increasingly larger and infinitely approaches a constant.

Proof. First, the probability $p(t)$ becomes increasingly larger. We use mathematical induction to prove this theorem. According to the lemma.

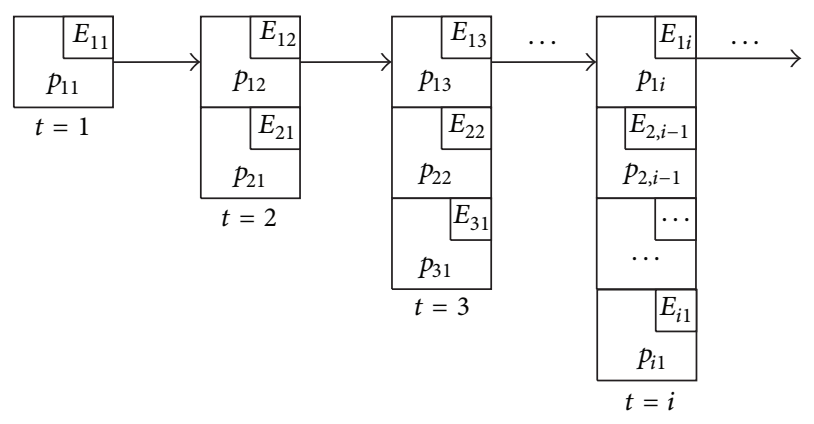

Figure 2: The probabilities that each part of the lurkers becomes spreaders; $p_{j h}$ represents the probability that lurkers $E_{j h}$ become spreaders.

For $i=1$,

$$
\begin{aligned}
p(2) & =\frac{p_{12} E_{12}+p_{21} E_{21}}{E_{12}+E_{21}}>\frac{p_{11} E_{12}+p_{11} E_{21}}{E_{12}+E_{21}} \\
& =\frac{p(1) E_{12}+p(1) E_{21}}{E_{12}+E_{21}}=p(1) .
\end{aligned}
$$

For $i=2$,

$$
\begin{aligned}
p(3) & =\frac{p_{13} E_{13}+p_{22} E_{22}+p(31) E_{31}}{E_{13}+E_{22}+E_{31}} \\
& >\frac{p_{13} E_{13}+p_{22} E_{22}+p_{11} E_{31}}{E_{13}+E_{22}+E_{31}} \\
& >\frac{p_{12} E_{13}+p_{12} E_{22}+p(1) E_{31}}{E_{13}+E_{22}+E_{31}} \\
& =\frac{p_{12}\left(E_{13}+E_{22}\right)+p(1) E_{31}}{\left(E_{13}+E_{22}\right)+E_{31}}=p(2) .
\end{aligned}
$$

For $i=3$,

$$
\begin{aligned}
p(4) & =\frac{p_{14} E_{14}+p_{23} E_{23}+p_{32} E_{32}+p_{41} E_{41}}{E_{14}+E_{23}+E_{32}+E_{41}} \\
& >\frac{p_{14} E_{14}+p_{23} E_{23}+p_{32} E_{32}+p_{11} E_{41}}{E_{14}+E_{23}+E_{32}+E_{41}} \\
& >\frac{p_{13} E_{14}+p_{13} E_{23}+p_{22} E_{32}+p(1) E_{41}}{\left(E_{14}+E_{23}\right)+E_{32}+E_{41}} \\
& >\frac{p_{13}\left(E_{14}+E_{23}\right)+p_{22} E_{32}+p(1) E_{41}}{\left(E_{14}+E_{23}\right)+E_{32}+E_{41}}=p(3) .
\end{aligned}
$$

Assuming the result is true for $i=k$, that is,

$$
\begin{aligned}
p(k) & =\frac{p_{1, k} E_{1, k}+p_{2, k-1} E_{2, k-1}+p_{3, k-2} E_{3, k-2} \cdots+p_{k, 1} E_{k, 1}}{E_{1, k}+E_{2, k-1}+E_{3, k-2}+\cdots+E_{k, 1}} \\
& >p(k-1) .
\end{aligned}
$$


To establish the truth of $p(k+1)>p(k)$, we need to show that

$$
\begin{aligned}
p(k+1) & \\
& =\frac{p_{1, k+1} E_{1, k+1}+p_{2, k} E_{2, k}+p_{3, k-1} E_{3, k-1} \cdots+p_{k+1,1} E_{k+1,1}}{E_{1, k+1}+E_{2, k}+E_{3, k-1}+\cdots+E_{k+1,1}} \\
& >\frac{p_{1, k}\left(E_{1, k+1}+E_{2, k}\right)+p_{2, k-1} E_{3, k-1} \cdots+p(1) E_{k+1,1}}{\left(E_{1, k+1}+E_{2, k}\right)+E_{3, k-1}+\cdots+E_{k+1,1}} \\
& =p(k) .
\end{aligned}
$$

Consequently, with an increase in the time step $t$, the probability $p(t)$ that a lurker becomes a spreader increases gradually because of the cumulative effect of memory. The probability is that the number of contact times infinitely approaches a constant [16]. Therefore, $p(t)$ infinitely approaches a constant when accumulated memories achieve a certain degree.

In light of above analysis, the probability $p(t)$ that lurkers become spreaders affected by memory accumulation at $t$ th time step is

$$
p(t)=(p-q) e^{-c(t-1)}+q
$$

where $p, q$, and $c$ are parameters. These three parameters reflect the characteristics of the variable memory effects rate. $p$ is the initial value of the memory effects function at $t=1$. The parameter $p$ reflects the importance of an event triggering rumors in the spreading process, and it is the initial probability that an individual becomes a spreader. A larger value for $p$ means that the spreaders more easily remember the rumor because the event is probably more important. $q \leq$ $1-p_{2}, q \in(0,1)$, is the maximal transformation probability. As time passes, $p(t)$ infinitely approaches $q$. The parameter $c$ can be regarded as the memory speed; namely, $c$ captures how quickly $p(t)$ reaches the maximum value $q$. The memory effects rate $p(t)$ is a probability varying over time $t$. Here, we do not consider interest decay and assume that the time scale for the rumor spreading is much faster than the memory decay. Figure 3 shows the transformation probability $p(t)$ as a function of $t$, given a different $c$ and a fixed $p_{2}=0.1$, $p(1)=0.05$, as shown in Figure 2 .

2.2. Rumor Spreading Model. Denote by $S(t), E(t), I(t)$, and $R(t)$ the density of the unaware, lurkers, spreaders, and stiflers at time $t, S(t)+E(t)+I(t)+R(t)=1$. As shown in Figure 4, the rumor spreading rules can be summarized as follows.

(1) Everyone needs time to determine the authenticity of rumor, so an unaware becomes a lurker with a probability 1 when an unaware individual contacts a spreader. The contact probability $k$ is decided by the specific network topology. Therefore, the reduced speed of the unaware $d S(t) / d t$ is proportional to the

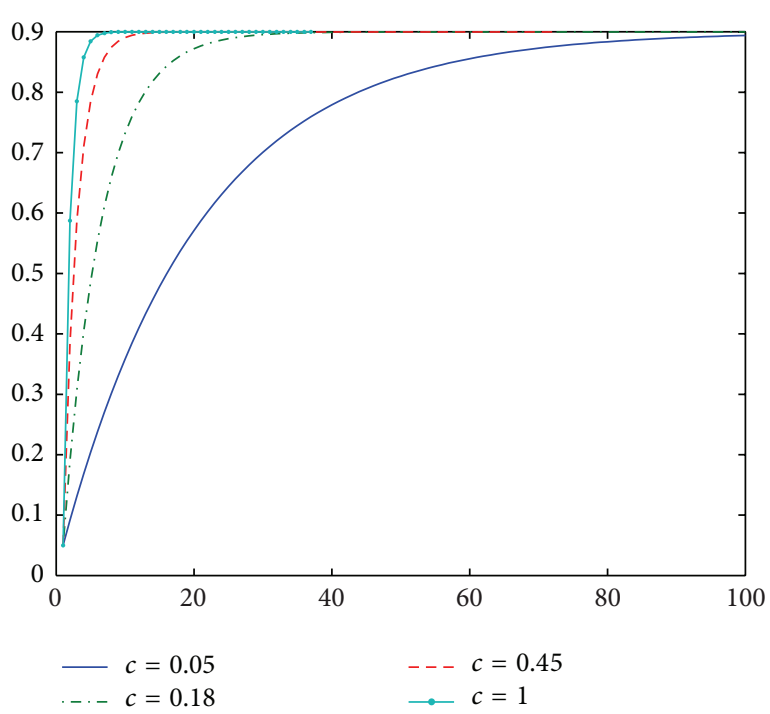

Figure 3: Memory effects function when $p_{2}=0.1, p(1)=0.1$.

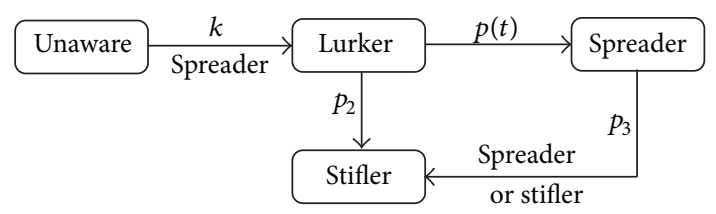

FIGURE 4: Structure of rumor spreading process.

number of the unaware $S(t)$ and the spreaders $I(t)$, so we can derive the differential equation:

$$
\frac{d S(t)}{d t}=-k S(t) I(t)
$$

(2) A lurker becomes a spreader at the rate of $p(t)$ and becomes a stifler at the rate of $p_{2}$, which depends on cognition. For example, some unaware individuals have strong knowledge structures and logical reasoning abilities, so they may have little interest in rumors; thus the probability $p_{2}$ is large. Because an unaware individual becomes a lurker with a probability 1 when an unaware contacts a spreader, the increased speed of the lurkers $d E(t) / d t$ is equal to

$$
\frac{d E(t)}{d t}=k S(t) I(t)-p(t) E(t)-p_{2} E(t) .
$$

(3) When two spreaders contact each other, both may find the two pieces of information inconsistent, so they stop the spread. When a spreader contacts a stifler, the spreader tries to stop the spread, as the stifler shows no interest in the rumor or denies its veracity. We suppose that the above cases occur at the same probability $p_{3}$ [17]. Therefore, the reduced speed of the spreaders $d I(t) / d t$ is proportional to the 
number of $I(t)$ and $R(t)+I(t)$, Additionally, a lurker becomes a stifler at the rate of $p(t)$, so we have

$$
\frac{d I(t)}{d t}=p(t) E(t)-k p_{3} I(t)(I(t)+R(t)) .
$$

(4) The increasing speed of the stiflers $d R(t) / d t$ is proportional to the number of existing $I(t)$ and $R(t)+I(t)$ from (3), and a lurker becomes a stifler at the rate of $p_{2}$ from (2), so we get

$$
\frac{d R(t)}{d t}=k p_{3} I(t)(I(t)+R(t))+p_{2} E(t) .
$$

Based on the previous discussion, the model is described using the following system of differential equations:

$$
\begin{aligned}
& \frac{d S(t)}{d t}=-k S(t) I(t), \\
& \frac{d E(t)}{d t}=k S(t) I(t)-p(t) E(t)-p_{2} E(t), \\
& \frac{d I(t)}{d t}=p(t) E(t)-k p_{3} I(t)(I(t)+R(t)), \\
& \frac{d R(t)}{d t}=k p_{3} I(t)(I(t)+R(t))+p_{2} E(t), \\
& \quad S(0)=S_{0}, \quad E(0)=0, \\
& I(0)=1-S_{0}>0, \quad R(0)=0 .
\end{aligned}
$$

2.3. Steady-State Analysis of Model. At the beginning of the rumor spreading process, the system has only the unaware and the spreaders and at the end has only the unaware and stiflers as an equilibrium state is reached. So here, we analyze the system's steady-state.

Substituting $I(t)+R(t)=1-S(t)-E(t)$ into differential equations (9), as the differential equations (7), (8), and (9) are not related to the $R(t)$, we only consider these three equations. Then the reduced limiting dynamic system is given by

$$
\begin{aligned}
& \frac{d S(t)}{d t}=-k S(t) I(t), \\
& \frac{d E(t)}{d t}=k S(t) I(t)-p(t) E(t)-p_{2} E(t), \\
& \frac{d I(t)}{d t}=p(t) E(t)-k p_{3} I(t)(1-E(t)-S(t)), \\
& S(0)=S_{0}, \quad E(0)=0, \quad I(0)=1-S_{0}>0 .
\end{aligned}
$$

From differential equations theory, systems (I) and (II) are homogeneous, which means that analyzing the properties of system (II) is equal to analyzing the properties of system (I). First, some definitions and the required theorem are introduced.

Our model is a nonautonomous differential dynamic system, the general form of which is

$$
\begin{aligned}
& \frac{d \mathbf{x}}{d t}=\mathbf{f}(t, \mathbf{x}), \\
& \mathbf{f}(t, 0)=0, \quad x \in R^{n} .
\end{aligned}
$$

Suppose that $I=\left[t_{0},+\infty\right], U=\{\mathbf{x} \mid\|\mathbf{x}\| \leq h\}$, and $V(t, \mathbf{x})$ is a continuous differentiable function defined in $I \times U . W(\mathbf{x})$ is a continuous differentiable function defined in $U$.

Definition 3 (see [18]). If $W(\mathbf{x})$ is a positive (negative) definite function, such that $V(t, \mathbf{x}) \geq W(\mathbf{x})(V(t, \mathbf{x}) \leq-W(\mathbf{x}))$ is true in $I \times U$ and $V(t, \mathbf{0})=0, V(t, \mathbf{x})$ is called a positive (negative) definite function in $I \times U$. If $V(t, \mathbf{x}) \geq 0(V(t, \mathbf{x}) \leq 0), V(t, \mathbf{x})$ is called a positive (negative) semidefinite function.

Definition 4 (see [18]). If $W_{1}(\mathbf{x})$ is a positive (negative) definite function, such that $|V(t, \mathbf{x})| \leq W_{1}(\mathbf{x})$, it is called $V(t, \mathbf{x})$ and has an infinitesimally small upper bound. If $W_{2}(\mathbf{x})$ is an infinite positive definite function, such that $V(t, \mathbf{x}) \geq$ $W_{2}(\mathbf{x})$, it is called $V(t, \mathbf{x})$ and has an infinite lower bound.

Theorem 5 (see [18]). Suppose that $V(t, \mathbf{x})$ is a positive definite function in $I \times R^{n}$, which has an infinitesimally small upper bound and an infinite lower bound and $\dot{V}(t, \mathbf{x})$ is a negative semi-definite, so the zero solutions for system (III) are global uniformly asymptotically stable.

Theorem 6. $P^{*}=\left(S^{*}, 0,0\right)\left(0 \leq S^{*}<1\right)$ is the equilibrium of the system (II).

Proof. Let the right side of each of the differential equations (12) be equal to zero in the system which gives the equation

$$
\begin{gathered}
-k S(t) I(t)=0, \\
k S(t) I(t)-p_{1} E(t)-p_{2} E(t)=0, \\
p_{1} E(t)-k p_{3} I(t)(1-E(t)-S(t))=0 .
\end{gathered}
$$

The feasible region for the equations is $R^{3}$, and so we study the equations in a closed set $A=\left\{(S, E, I) \in R^{3} \mid S+E+I \leq\right.$ $1, S, E, I \geq 0\}$.

From (11) and (12), we can get $\left(p(t)+p_{2}\right) E(t)=0$, so $E=0$. Substituting $E=0$ into (13), we have $k p_{3} I(t)(1-S(t))=0$. Adding (12), we can get $I(t)=0$. From (13), the system has the equilibrium $P^{*}=(S, E, I)=\left(S^{*}, 0,0\right)\left(0 \leq S^{*}<1\right)$.

Therefore, we can obtain the equilibrium for the system (I) $Q^{*}=\left(S^{*}, 0,0, R^{*}\right)$, where $S^{*}+R^{*}=1$; that is, the rumor must disappear with time, and all $S^{*}$ and $R^{*}$ that satisfy $S^{*}+R^{*}=1$ represent stable situations. Next, we look at the stability of the equilibrium $P^{*}=\left(S^{*}, 0,0\right)$.

Theorem 7. The equilibrium $P^{*}$ is globally uniformly asymptotically stable.

Proof. Let the Liapunov function be $V(S, E, I)=F(S)+$ $G(E)+H(I)$, and taking the derivative of $V$ versus $t$ along the solution for the equations, we have

$$
\begin{aligned}
V^{\prime}(S, E, I) \\
=-F^{\prime}(S) k S(t) I(t)+G^{\prime}(E) \\
\quad \times\left[k S(t) I(t)-p(t) E(t)-p_{2} E(t)\right] \\
\quad+H^{\prime}(I)\left[p(t) E(t)-k p_{3} I(t)(1-E(t)-S(t))\right] .
\end{aligned}
$$




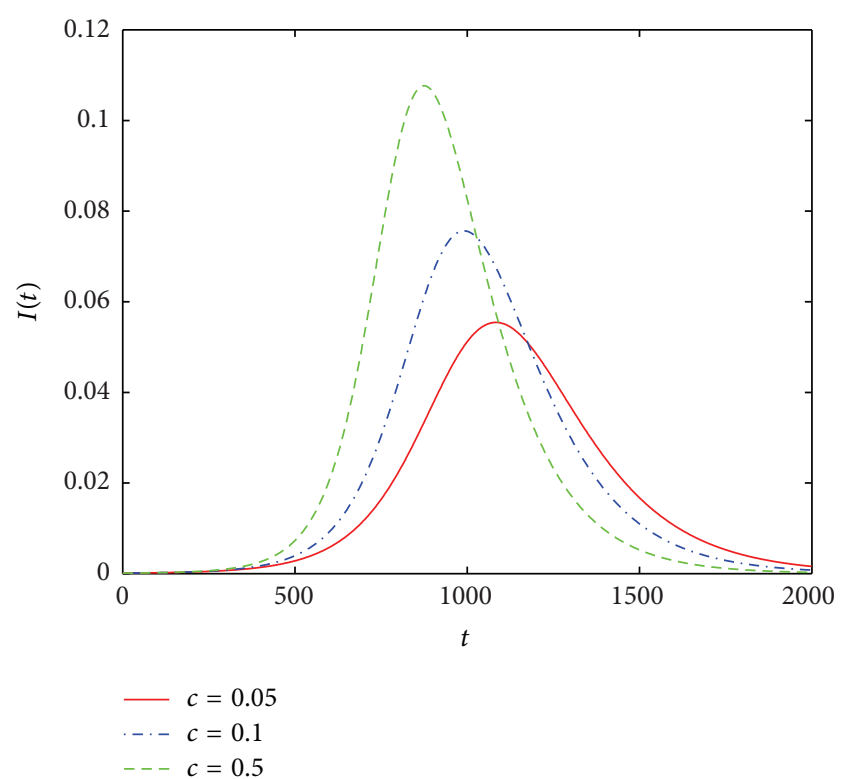

(a)

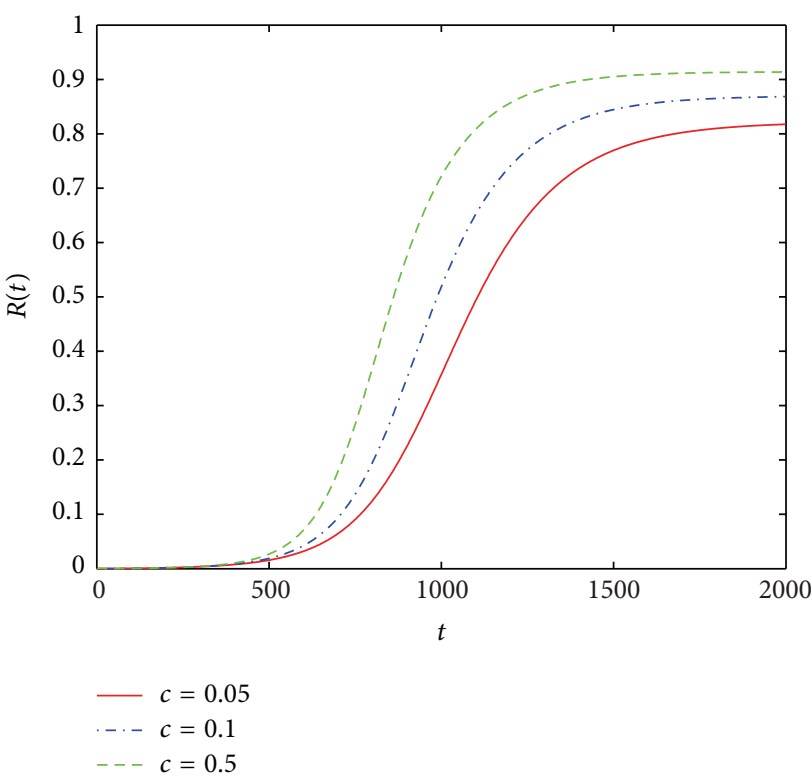

(b)

FIGURE 5: Density of spreaders and stiflers on regular networks.

In order to ascertain $V(S, E, I)$ and $V^{\prime}(S, E, I)$, and taking the values of $F^{\prime}(S)=1, G^{\prime}(E)=1$, and $H^{\prime}(I)=1$, we get $V(S, E, I)=S+E+I$, and

$$
\begin{aligned}
V^{\prime}(S, E, I)= & -k S(t) I(t)+k S(t) I(t)-p(t) E(t)-p_{2} E(t) \\
& +p(t) E(t)-k p_{3} I(t)(1-E(t)-S(t)) \\
= & -p_{2} E(t)-k p_{3} I(t)(1-E(t)-S(t)) \leq 0 .
\end{aligned}
$$

So we get $V(S, E, I)$, which is positive definite, $0 \leq$ $V(S, E, I) \leq 1+S$; that is, it has an infinitesimally small upper bound and an infinite lower bound, and $V^{\prime}(S, E, I)$ is negative semidefinite in the feasible region $A=\left\{(S, E, I) \in R^{3}\right.$ । $S+E+I \leq 1, S, E, I \geq 0\}$.

From Theorem 5 , the equilibrium $P^{*}$ is globally uniformly asymptotically stable.

\section{Numerical Analysis}

In the following sections, simulations are conducted using the Runge-Kutta method and MATLAB in artificial networks and online social networks, respectively. First, we examine how the proportion of spreaders and stiflers changes over time by varying the function parameters. Then, we discuss the effect of the average degree of networks on rumor spreading. Finally, numerical solutions for the established model are conducted on the Sina Microbolg online social network, and we make comparisons between a constant versus a variable memory rate on the Sina Microbolg.

3.1. Sensitivity Analysis in Artificial Networks. To compare the results, the proposed model was performed on two artificial networks with different values for parameter $c$. We chose regular networks and ER random networks with the same network size $N=10000$ and the same average degree $\langle k\rangle=$ 16.

(1) Regular networks [19]: this is a nearest-neighbor coupled network, in which every node is of the same degree.

(2) Random networks [20]: two different nodes are connected with a probability of $P=0.0016$, so we get random networks with $N$ nodes and $P N(N-1) / 2$ edges. ER random networks degree distributions are an approximate Poisson distribution, $\langle k\rangle=P(N-$ $1) \approx P N$. This distribution reaches its peak value at the average degree $\langle k\rangle$ [21].

(3) BA network [22]: the network exhibits a power-law degree distribution $p(k) \sim k^{-\gamma}$ with $\gamma=3$.

Given that the other parameters are fixed, we compared the rumor spreading processes on the three networks with different values for $c$. The smaller the value of $c$, the slower the speed of increase for $I(t)$. So here, we set $c=0.05, c=0.1$, and $c=0.5$. Figure 5 illustrates how the density of spreaders and stiflers changes over time for the different values of $c$ in a regular network. From a macroscopic perspective, we found that the greater parameter $c$ is, the higher the peak for $I(t)$ is, and, as parameter $c$ increases, the size of the stiflers increases, as larger $c$ indicates a higher speed for $p(t)$ to reach the maximum value $q$. Figure 5(a) describes how the density of spreaders changes with changes in parameter $c$. The green line indicates a scenario in which the memory speed is very fast. We have carefully checked that the results are not sensitive to $c$ when $c \geq 0.5$. It can be seen that the higher parameter $c$ is, the larger the spreader peak value is and the slower the rumor terminates. Figure 5(b) describes how the density of stiflers 


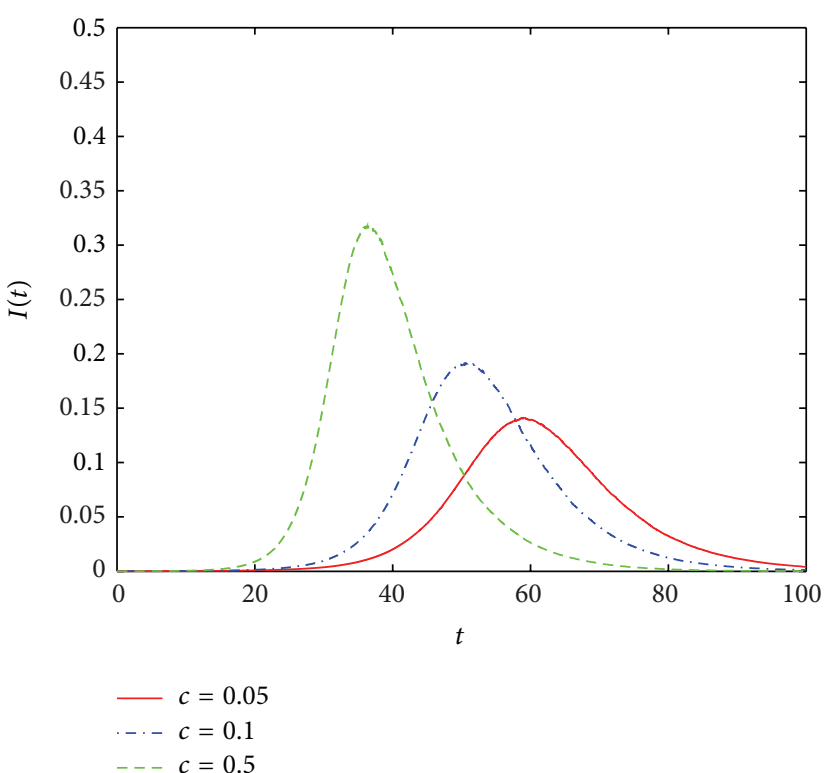

(a)

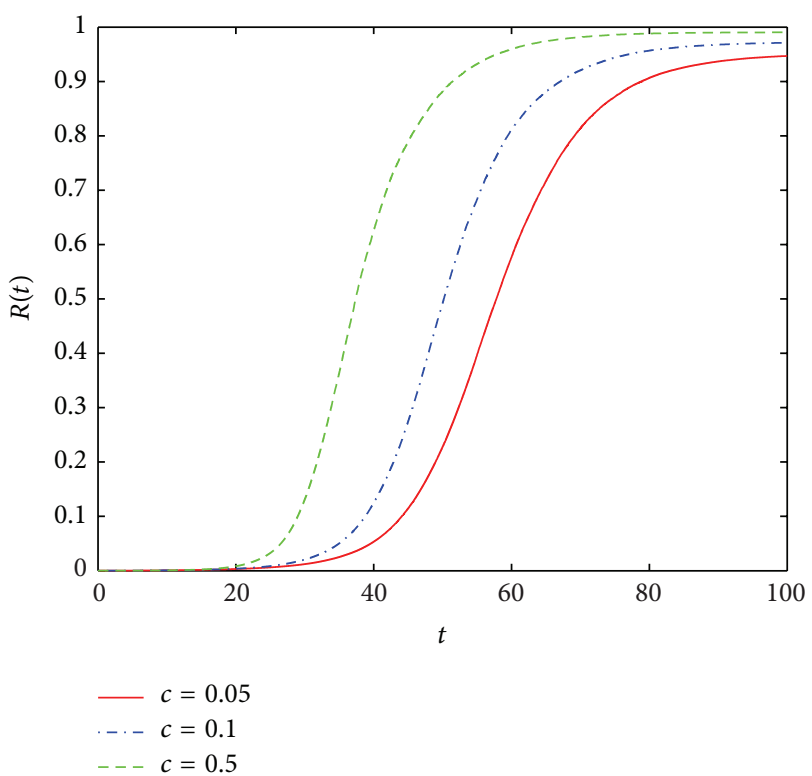

(b)

FIgURE 6: Density of spreaders and stiflers on random networks.

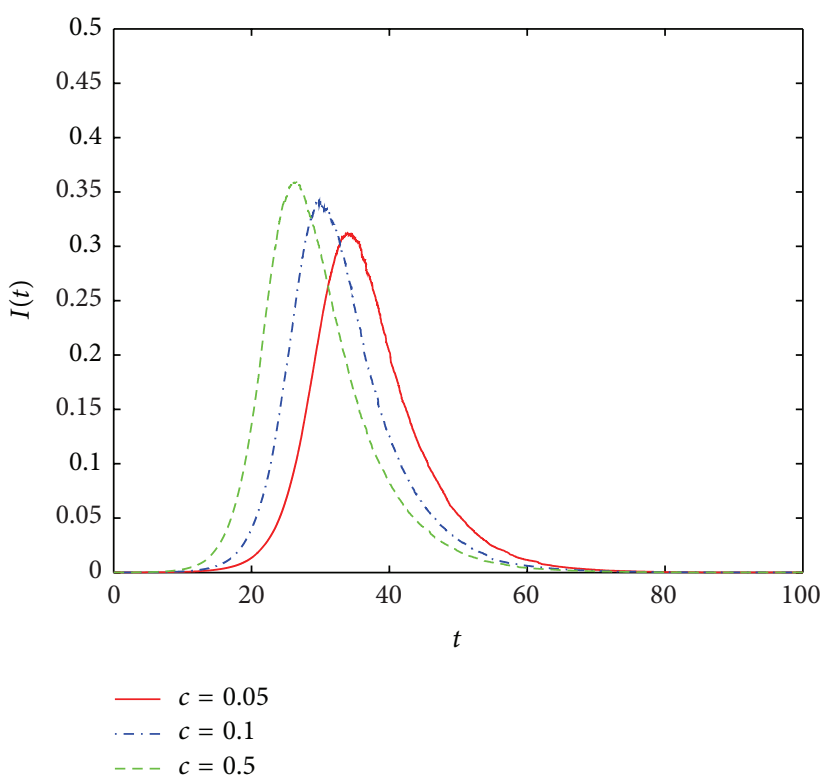

(a)

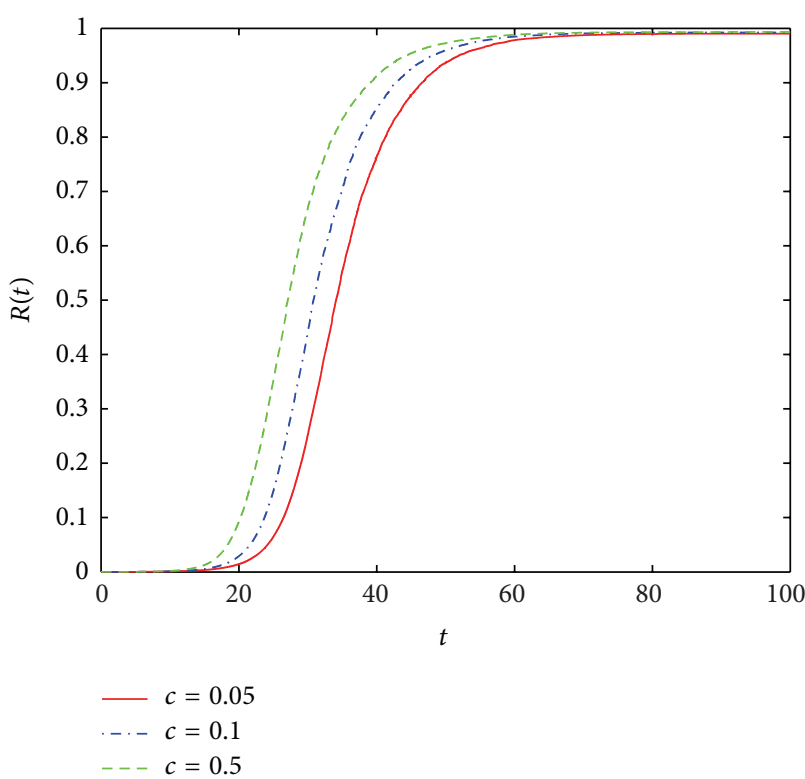

(b)

FIGURE 7: Density of spreaders and stiflers on BA networks.

changes with $c$ over time. The final value for the stifler density $R(t)$ is greater, which indicates the number of people affected by a rumor when $c$ is larger. Clearly, the larger the value of $c$ when other parameters are fixed, the broader the rumor's influence. A larger $c$ indicates that the $p(t)$ speed is higher and reaches the maximum value, less spreaders change into stiflers, and therefore the influence of the rumor increases.

Figures 6 and 7 illustrate how the density of spreaders and stiflers changes over time with different values for $c$ in ER random networks and BA networks. Similar to the regular network, it can be seen that rumors spread more broadly when $c$ is larger. However, there is a most significant impact from the importance of events on rumor spreading in random networks. That is to say, rumor spreading is sensitive to parameter $c$, which is related to the topology of random networks. From Figure 7(a), in the same situation, if $c$ is larger, the rumors spread most quickly in BA networks than in the other two networks. And the number of $I(t)$ follows the relation $\mathrm{BA}>$ random $>$ regular. These results are consistent with the traditional understanding of epidemic 


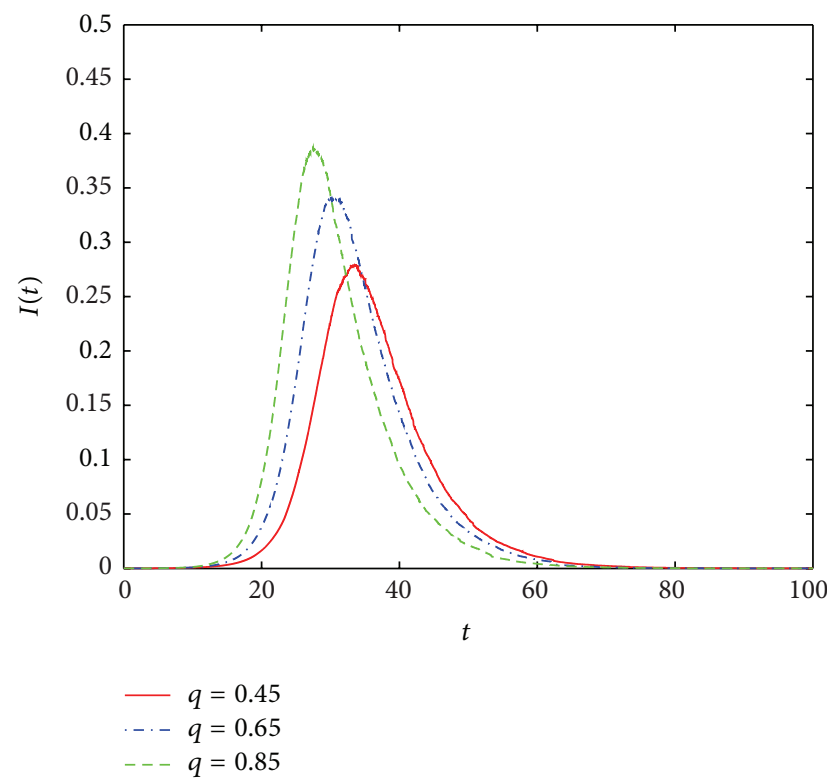

(a)

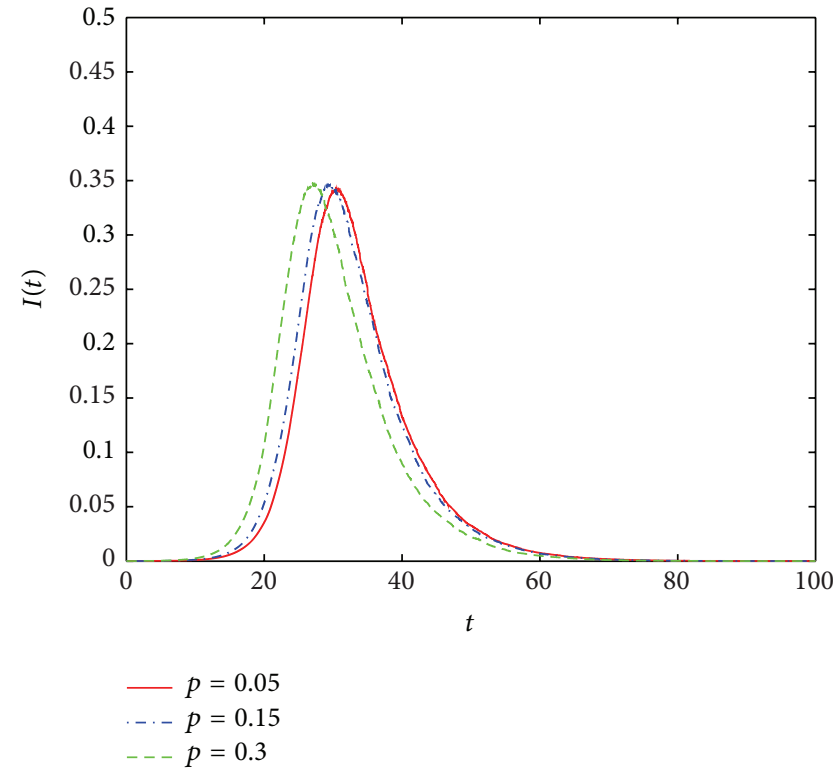

(b)

FIGURE 8: Density of spreaders with different $p$ and $q$ on BA networks.

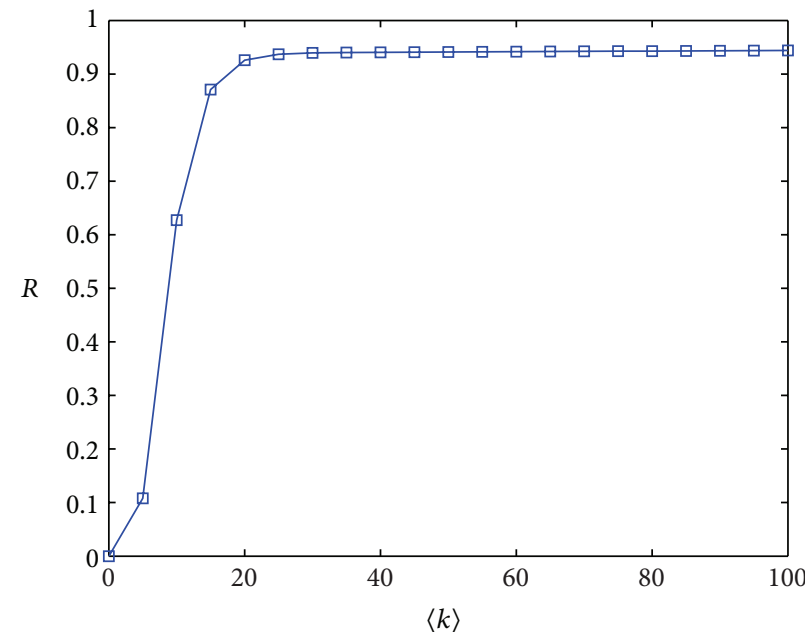

(a)

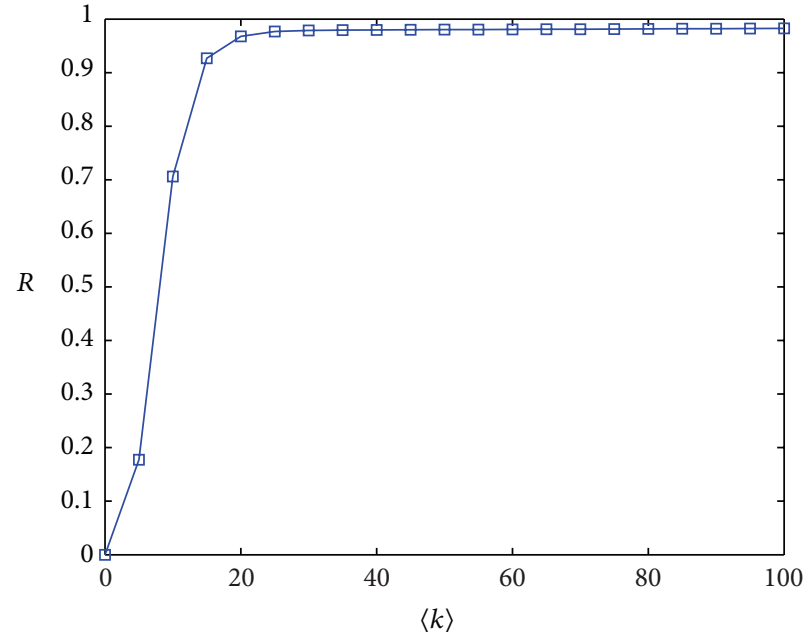

(b)

FIGURE 9: Density of stiflers on regular networks and random networks.

and information spread in that the speed and range of the spread obey the relationship [8]. Figure 7(b) describes how the density of stiflers changes as $c$ changes over time. When parameter $c$ is fixed, we can see that the rumor spreads faster and the final size of the stiflers, $R(t)$, is largest in BA networks. In other words, rumors spread faster and more broadly on BA networks than the other networks. For all the networks, the bigger the value of $c$, when other parameters are fixed, the bigger the rumors influence.

Because BA networks are closer to real social networks, we analyze the effect of the parameters $p$ and $q$ on these networks, respectively. Figure 8(a) illustrates how the spreader density changes as parameter $q$ changes in BA networks.
Given that other parameters are fixed, the bigger the value of $q$, the bigger the rumor's influence. $q$ is the maximal transformation probability that lurkers become spreaders; in reality, along with the increase of $q$, there are more lurkers becoming spreaders. As a result, the number of spreaders increases, which increases the influence of the rumor. Figure 8(b) describes how the density of spreader changes as $p$ changes over time. Also, we can see in Figure 8(b) that the bigger the value of $p$, when other parameters are fixed, the bigger the rumor's influence. $p$ is the initial probability that an individual becomes a spreader. In reality, a large $p$ means that the individual thinks that an event triggering rumors is very important in the spreading process, which can make more 


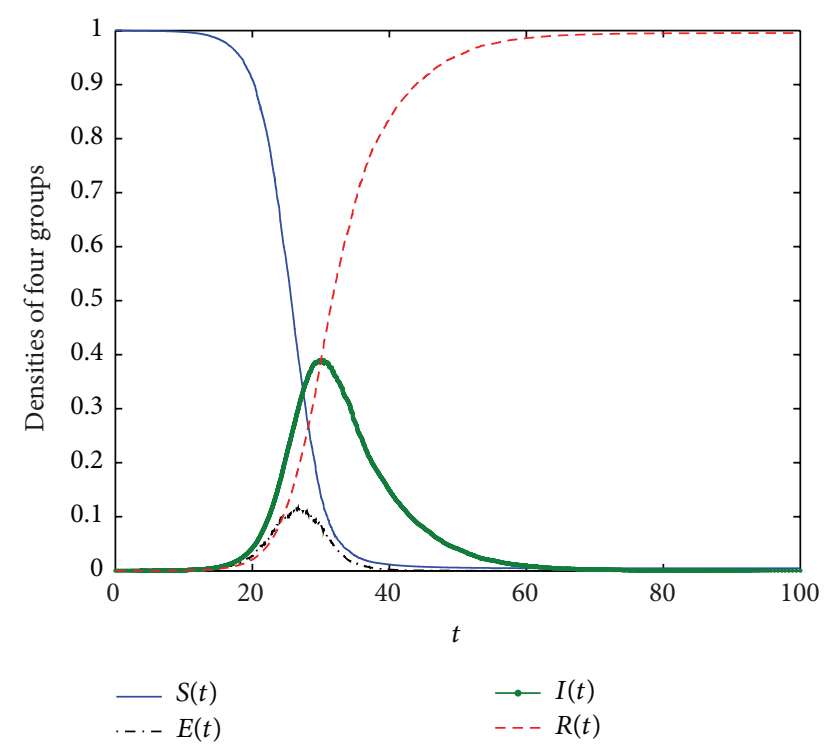

FIGURE 10: Densities of unaware individuals, spreaders, lurkers, and stiflers over time with $c=0.1, p(1)=0.05, q=0.65, p_{2}=0.15$, and $q=0.17$.

individuals change into spreaders and, therefore, increase the influence of the rumor. But $p$ has little influence on the spread of rumor.

Figure 9 displays the final spread size for regular networks and random networks as a function of the average degree $\langle k\rangle$ when $c=0.1$. In random networks, because $\langle k\rangle=$ $P(N-1) \approx P N$, analyzing the effect of average degree $\langle k\rangle$ is equal to analyzing the effect of connected probability $P$ on random network when network size is fixed. There are many similarities between Figures 9(a) and 9(b). It is obvious that $R$ increases with $\langle k\rangle$, but the final spreading size is larger in random networks at same average degree. Moreover, the curves show that $R$ peaks suddenly with small $\langle k\rangle$, which means that $R$ is sensitive when $\langle k\rangle$ is small. Rumor spreading enhancement with the average degree could be more significant in random networks than that on regular networks when $\langle k\rangle$ is small because of the different network topologies, while for a large $\langle k\rangle$, the changing scale for $R$ is smaller, which means that the final size of $R$ is not sensitive to the average degree when $\langle k\rangle$ is large. We have carefully checked the fact that, with an increase in $\langle k\rangle, R$ becomes increasingly larger and is finally equal to 1 when $\langle k\rangle$ is large enough. That is, when all individuals change to stiflers, the whole population is infected. In general, the final size of $R$ is sensitive to $\langle k\rangle$ when $\langle k\rangle$ is small but not sensitive to $\langle k\rangle$ when $\langle k\rangle$ is large.

3.2. Rumor Spreading in Social Networks. To study how rumors spread on social networks, we simulated the rumor spreading process on a snapshot of the China's largest microblog site, Sina Microblog networks which had been crawled by Yuan and Liu [23]. This snapshot consisted of 118,517 nodes and 2728,213 edges, with the degree distribution obeying the power law $p(k) \sim k^{-\gamma}$ [24], $\gamma=1.43$. We chose online social networks because of the available network data and because we feel that their structure might be similar to that of other real world social networks.

Figure 10 corresponds to a case where $P=0.05, q=$ 0.65 , and $c=0.1$, which shows the general trends for the four kinds of agents in our rumor spreading model. From the simulation, we find that as the rumor propagates at the beginning of the process, there is a sharp increase in the number of spreaders. With the further spreading of the rumor, the number of spreaders quickly reaches a peak at about $t=30$ and then decrease as time goes on. Finally, the number of spreaders is zero and the spreading of the rumor terminates. With an increase in time $t$, the number of unaware individuals $S(t)$ always reduces while the number of stiflers $R(t)$ always increases, finally reaching a balance at the end of the rumor spreading process. From this figure, it can be seen that almost all individuals become stiflers after about 70 time steps. The variation trend for the number of lurkers is similar to that of the spreaders, in that they increase at first and then decrease to zero. However, this increasing and decreasing trend for the lurkers is much more moderate than for the spreaders, and, as can be seen, the peak for the lurkers is much smaller than that for the spreaders.

Figure 11 shows how the densities of spreaders and stiflers change over time for variable and constant memory rate in the Sina Microbolg network. For the variable rate, it can be seen that the peak of the spreader is much higher, compared to the constant rate case. It can be seen from Figure 11(b) that final stifler density value $R(t)$ is greater, which means that the population affected by the rumor is greater under a variable memory rate. At the same time, the rumor terminates faster when the memory rate is nonconstant, which shows that the addition of function $p(t)$ has accelerated the pace of the entire rumor spreading process. Generally speaking, the rumor spreads faster and more broadly under variable rates on the Sina Microbolg. In real social networks, the number of people who spread rumors is small, but the rumors can spread widely as social networks have a high clustering coefficient which means the number of the lurkers who come into contact with the rumor is greater. Rumors spread quickly in the early stages through the friends of the people on social networks, and, some time later, the number of people who come into contact with the rumor reaches a maximum, after which people lose interest because local authorities have debunked the rumor or people have an increased awareness of the truth or otherwise of the rumor. Thus the speed of the transmission reduces. Finally, the spreaders gradually disappear, and the entire population becomes stiflers.

\section{Discussion}

In our opinion, the cumulative effects of memory play an important role in the rumor spreading process. Although some variants of the SIR model do consider the memory effects, they do not consider the cumulative memory effect. That is to say, they reflect the memory effects using a constant parameter rather than a variable parameter which changes with time across the entire rumor spreading process. In order 


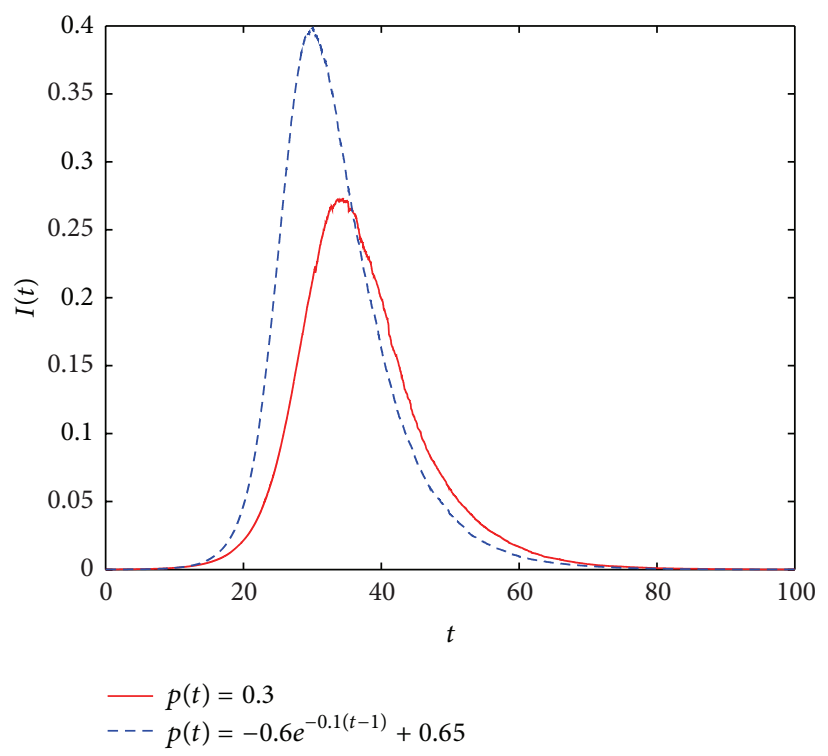

(a)

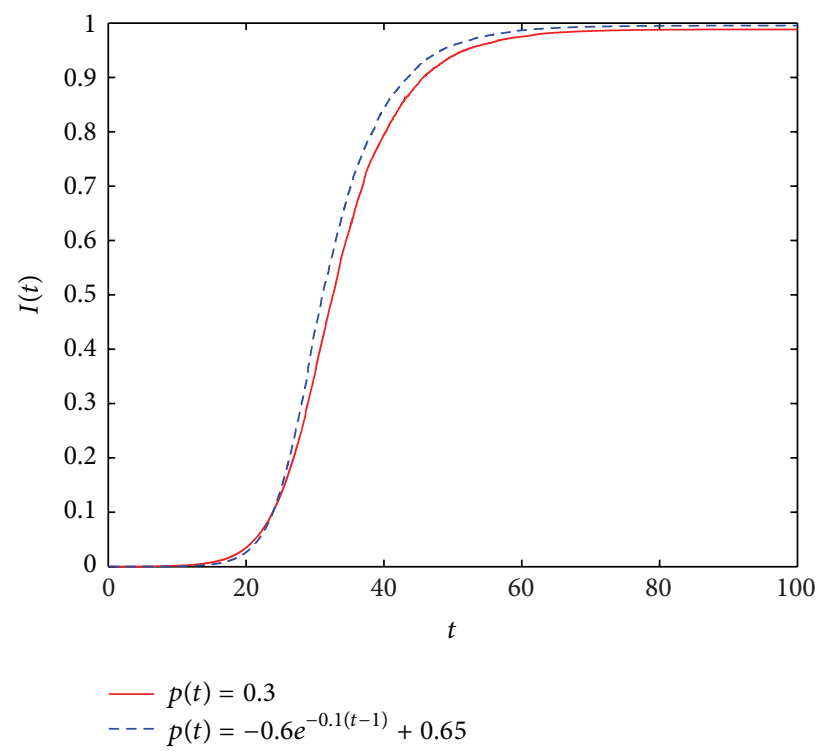

(b)

FIGURE 11: Density of spreaders and stiflers on Sina Microblog network for the values of the memory rate is variable $\left(p(t)=-0.6 e^{-0.1(t-1)}+0.65\right)$ and constant $(p(t)=0.3)$, respectively.

to better describe the rumor spreading process, we establish mean-field equations with variable memory effects rates in the network. As such, previous models which consider the memory effects rate as a constant become a special case of the new model. We compared our new rumor spreading model to the traditional model on an online social network. The simulation results showed that the function $p(t)$ induced a broader and faster rumor spreading process. Although rumor spreading simulates on a given snapshot of the Sina Microblog networks. The model does not take into account all rumor spreading features, such as the forgetting mechanism [25], or the nonredundancy of contact [16], but it largely reflects the cumulative memory effects in the variants of the SIR model. We also found that rumor spreading is more influenced by parameter $c$ in random networks, which is caused by the specific topology of random networks. At the same time, it can be seen that the random networks are more effective in rumor spreading which supports Zanette's experiment. In addition, our experiments show that a high average degree is very helpful in promoting the spreading of rumor. This is expected because as the average degree of networks increases, there are more friends for a user, which means there are more chances for spreaders to contact the unaware, making the spreading of the rumor much easier. However, the final number of people affected by the rumors is sensitive to the average degree $\langle k\rangle$ when $\langle k\rangle$ is small, but for a large $\langle k\rangle$, nearly all individuals spread the rumor. In fact, the entire population knows the rumor quickly when $\langle k\rangle$ reaches a certain level, at which point the increased average degree has no effect.

From the discussion of the cumulative effects of memory in this paper, governments should use this special property to control the spread of rumors. Because a sustained and rapid spread of information deepens people's impression about this information and to a certain extent improves the credibility of the information, when an emergency arises, governments should release official information at a high frequency to ensure the sustained and rapid spreading of official information, which may help avoid the spread of panic and prevent the spread of unfounded rumors.

\section{Conclusions}

In this paper, we have proposed a rumor spreading model which considers changing memory effects over time. At the same time, the speed that $p(t)$ takes to reach a maximum value and the importance of an event were considered. The cumulative effect of memory reflects the repeatability of the spreading characteristics of the rumor, so the probability $p(t)$ of a lurker becoming a spreader becomes increasingly larger and infinitely closer to a constant. Therefore, the probability is not a constant but a function of time in entire rumor spreading process. This conclusion was demonstrated in this study. We established a function $p(t)$ to describe the cumulative effect of memory, and this function as a parameter was added to the rumor spreading model, and a dynamic analysis of the model was conducted.

The simulations showed how the memory effects do affect rumor spreading when the parameters of the memory function $p(t)$ vary in regular networks, ER random networks, and BA networks. The simulation results indicated that a rumor spreads faster and more broadly in three network types when the initial memory rate $p$ is larger; the larger the maximum value of the memory rate $q$, the faster the memory speed $c$, and the greater the peak value for the spreaders. These results suggest that rumor spreading is sensitive to 
parameter $c$ in random networks, BA networks are most effective for rumor spreading, and the initial memory rate has little influence on the spread of rumor. We conducted a sensitivity analysis on the average degree in our model, the results of which demonstrated that the average degree plays a positive role in promoting rumor spreading. The final number of people affected by the rumors was found to be sensitive to the average degree $\langle k\rangle$ when $\langle k\rangle$ is small. Finally, the numerical solutions also demonstrated that the spreader peak value and final rumor spreading size on the Sina Microblog were much higher when the memory effects rate changes over time, compared to the case when the memory effects rate is considered a constant. Further study will be conducted which includes the other differences between rumor spreading and epidemic spreading. Further, investigation of rumor control strategies in different network topologies is also a future research direction.

\section{Conflict of Interests}

The authors declare that there is no conflict of interests regarding the publication of this paper.

\section{Acknowledgments}

This research was supported by the Major Bidding Program of the National Social Science Foundation of China (Grant nos. 12 and ZD217) ad the National Natural Science Foundation of China (Grant no. 71301109) and supported by the Natural Science Foundation of Anhui Province (Grant no. KJ2011B140). We appreciated all support in finance and in spirit.

\section{References}

[1] W. A. Peterson and N. P. Gist, "Rumor and public opinion," American Journal of Sociology, vol. 57, no. 2, pp. 159-167, 1951.

[2] D. J. Daley and D. G. Kendall, "Epidemics and rumours," Nature, vol. 204, no. 4963, article 1118, 1964.

[3] R. M. Anderson, R. M. May, and B. Anderson, Infectious Diseases of Humans: Dynamics and Control, vol. 28, Wiley, 1992.

[4] H. W. Hethcote, "The mathematics of infectious diseases," SIAM Review, vol. 42, no. 4, pp. 599-653, 2000.

[5] A. Sudbury, "The proportion of the population never hearing a rumour," Journal of Applied Probability, vol. 22, no. 2, pp. 443446, 1985.

[6] L. J. Zhao, H. X. Cui, X. Y. Qiu, X. Wang, and J. Wang, "SIR rumor spreading model in the new media age," Physica A, vol. 392, no. 4, pp. 995-1003, 2013.

[7] M. Nekovee, Y. Moreno, G. Bianconi, and M. Marsili, “Theory of rumour spreading in complex social networks," Physica A: Statistical Mechanics and its Applications, vol. 374, no. 1, pp. 457470, 2007.

[8] J. Wang, L. Zhao, and R. Huang, "SIRaRu rumor spreading model in complex networks," Physica A, vol. 398, no. 15, pp. 4355, 2014.

[9] S. H. Strogatz, "Exploring complex networks," Nature, vol. 410, no. 6825 , pp. 268-276, 2001.

[10] C. Liu and Z.-K. Zhang, "Information spreading on dynamic social networks," Communications in Nonlinear Science and Numerical Simulation, vol. 19, no. 4, pp. 896-904, 2014.
[11] S. Han, F. Z. Zhuang, Q. He, Z. Shi, and X. Ao, "Energy model for rumor propagation on social networks," Physica A: Statistical Mechanics and Its Applications, vol. 394, no. 15, pp. 99-109, 2014.

[12] P. S. Dodds and D. J. Watts, "Universal behavior in a generalized model of contagion," Physical Review Letters, vol. 92, no. 21, Article ID 218701, 2004.

[13] L. Zhao, J. Wang, Y. Chen, Q. Wang, J. Cheng, and H. Cui, "SIHR rumor spreading model in social networks," Physica A: Statistical Mechanics and its Applications, vol. 391, no. 7, pp. 2444-2453, 2012.

[14] L. J. Zhao, X. Y. Qiu, X. L. Wang, and J. Wang, "Rumor spreading model considering forgetting and remembering mechanisms in inhomogeneous networks," Physica A, vol. 392, no. 4, pp. 987994, 2013.

[15] D. Benjamin, F. Mahmoud, and F. Tobias, "Experimental analysis of rumor spreading in social networks," in Design and Analysis of Algorithms, vol. 7659 of Lecture Notes in Computer Science, pp. 159-173, Springer, Berlin, Germany, 2012.

[16] L. Lü, D.-B. Chen, and T. Zhou, "The small world yields the most effective information spreading," New Journal of Physics, vol. 13, Article ID 123005, 2011.

[17] L. Huo, P. Huang, and C.-x. Guo, "Analyzing the dynamics of a rumor transmission model with incubation," Discrete Dynamics in Nature and Society, vol. 2012, Article ID 328151, 21 pages, 2012.

[18] Z. E. Ma and Y. C. Zhou, The Qualitative and Stability Theory of Ordinary Differential Equations, Science Press, Beijing, China, 2001, (Chinese).

[19] D. J. Watts and S. H. Strogatz, "Collective dynamics of 'smallworld' networks," Nature, vol. 393, no. 6684, pp. 440-442, 1998.

[20] P. Erdös and A. Rényi, “On random graphs," Publicationes Mathematicae Debrecen, vol. 6, pp. 290-297, 1959.

[21] B. Bollobás, Random Graphs, Academic Press, New York, NY, USA, 2001.

[22] A. L. Barabási and R. Albert, "Emergence of scaling in random networks," Science, vol. 286, no. 5439, pp. 509-512, 1999.

[23] W. Yuan and Y. Liu, "Empirical analysis and evolution modeling of network topological structure in microblog," in Proceedings of the 5th IEEE International Conference on Intelligent Networking and Collaborative Systems (INCoS '13), pp. 656-661, Xian, China, September 2013.

[24] A. Clauset, C. R. Shalizi, and M. E. Newman, "Power-law distributions in empirical data," SIAM Review, vol. 51, no. 4, pp. 661-703, 2009.

[25] J. Gu, W. Li, and X. Cai, "The effect of the forget-remember mechanism on spreading," European Physical Journal B, vol. 62, no. 2, pp. 247-255, 2008. 


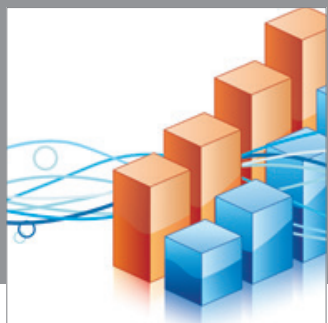

Advances in

Operations Research

mansans

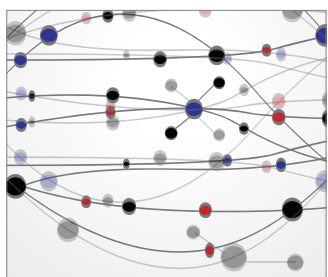

The Scientific World Journal
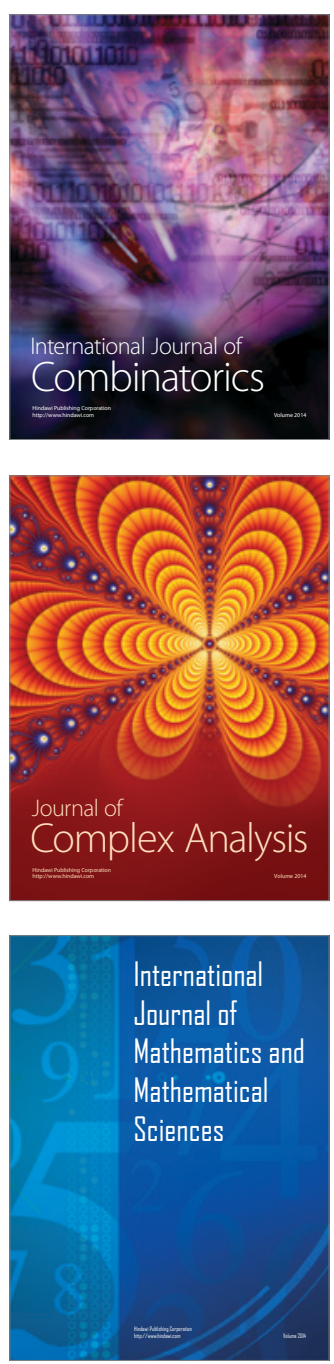
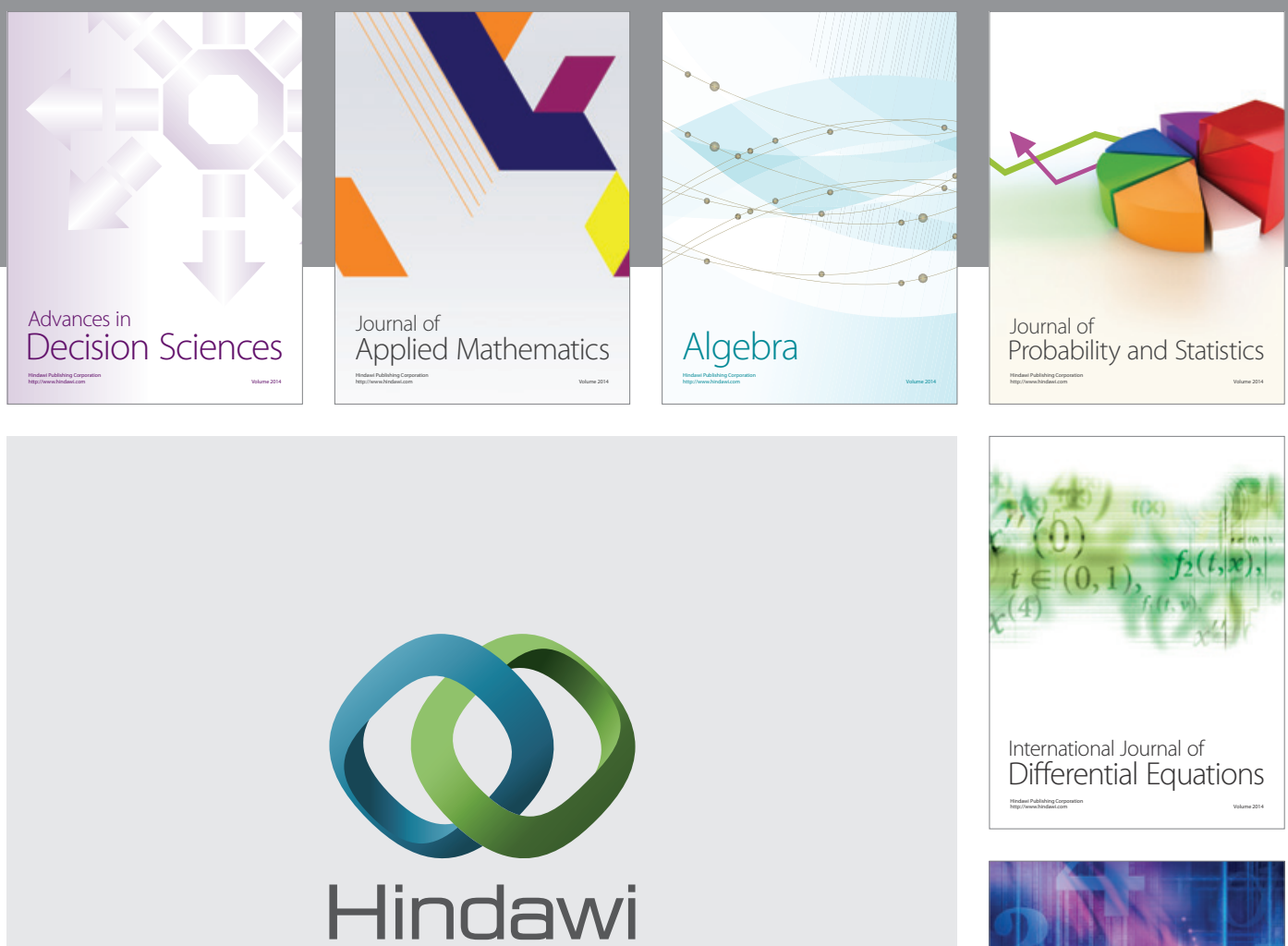

Submit your manuscripts at http://www.hindawi.com
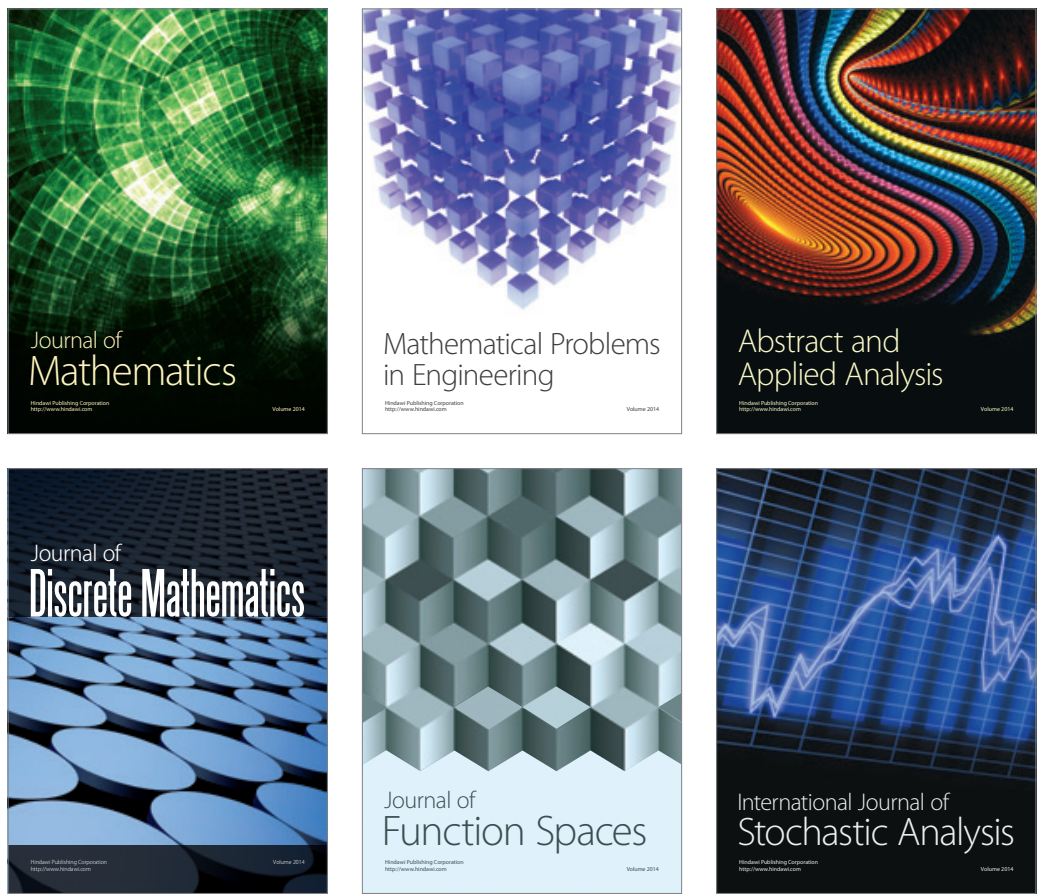

Journal of

Function Spaces

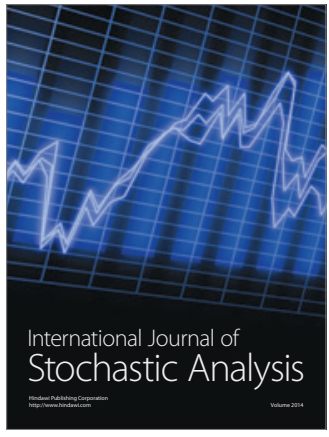

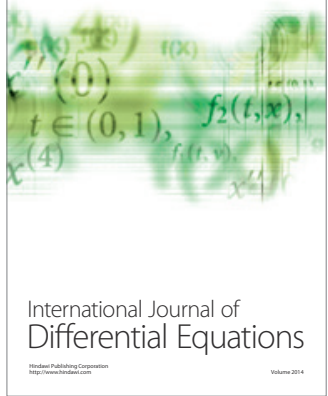
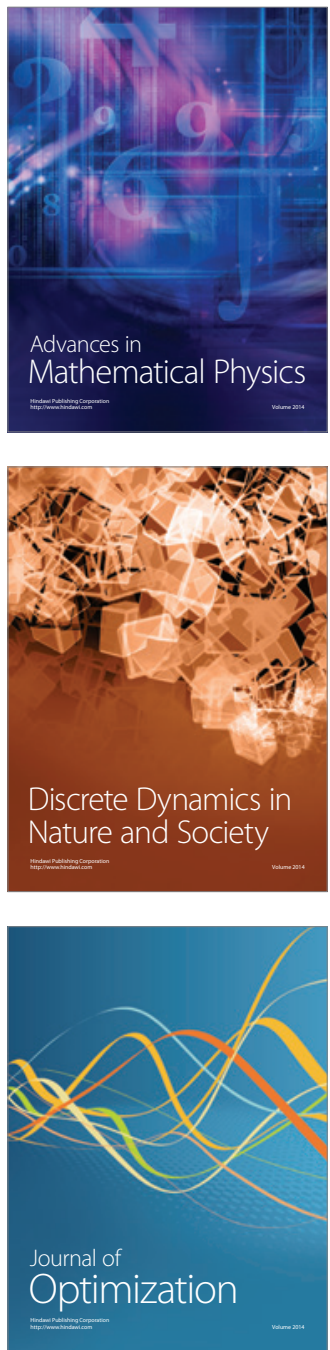